\title{
Two Fuzzy Controllers Alternating for Cartpole System
}

\author{
Sunggyu Kwon \\ Faculty of Mechanical and Automotive Engineering \\ Keimyung University, Daegu, Korea
}

\begin{abstract}
A control system composed of two fuzzy controllers is proposed to balance the pole as well as to move the cart to the center of the track of the cartpole system. The two fuzzy controllers are designed with 2 input variables respectively and their control characters are studied in order to devise a control scheme that alternates the two fuzzy controllers. It is found that the control system using the scheme works well even though there is some residual oscillations of the pole and the cart.
\end{abstract}

Key Words : Cartpole system, fuzzy control, inverted pendulum. cooperative fuzzy control

\section{Introduction}

Control of the cartpole [1] system has been the object of many studies in the literature of control and neural networks. Figure 1 shows the cartpole system composed of a rigid pole and a cart. The pole is hinged on the center of the top of the cart that travels along a track of limited length. Thus, the pole can rotate around the pivot in the vertical plane of the cart. The state of the cartpole system is defined by 4 state variables: $x$ and $\dot{x}$, the position and the velocity of the cart and $\phi$ and $\dot{\phi}$, the angular position and the velocity of the pole. An impulsive force is applied to the cart at discrete time intervals to control the system. The goal of the control is to make the pole upright and regulate the cart back to the center of the track.

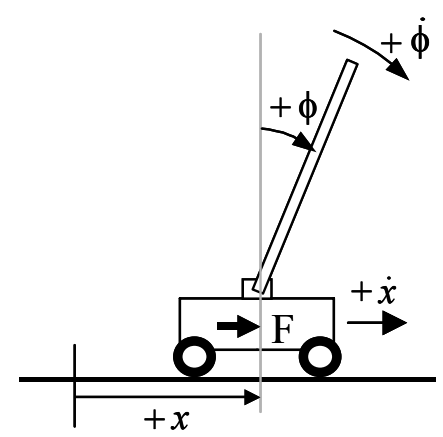

Figure 1. A cartpole system

Some control tasks of the cartpole system are simply to balance the pole without considering the position of the cart. Lee [2] developed an intelligent control scheme by integrating fuzzy control and reinforcement learning techniques. To balance the pole, 7 linguistic labels for the pole angle and 3 labels for the angular velocity of the pole were employed to generate continuous forces as output. Deng [3] proposed a neural-fuzzy BOXES [4] control system with reinforcement

Manuscript received Jan. 29. 2008; revised May. 27. 2009. learning where the state space is divided into some overlapping fuzzy boxes by defining input membership functions for each state variable.

For balancing as well as centering, some fuzzy control techniques make use of the characteristics of the cartpole control. Lin and Sheu [5] proposed a hybrid-control that combines a fuzzy logic-control and a linear state feedbackcontrol for a pendulum-car system. In order to improve the unstable control phenomenon within a limited length of car position, the fuzzy controller was to swing up the pole and the linear state feedback controller was to stabilize the cartpole system when the pendulum is near its upright position. For a switching-type sliding mode control, Li and Shieh [6] divided the dynamic response of the cartpole system into approach and departure mode and two types of fuzzy sliding mode controller designed for the two modes. The control scheme was to make the cartpole system in departure mode first and then to make the system in approach mode by switching two types of the controllers. Yi and Yubazaki [7] presented a fuzzy controller for stabilization control of inverted pendulum system where the fuzzy controller was composed of 4 input state variables and 1 output variable. Each input variable is given with a single input rule module (SIRM) and a dynamic importance degree. The parameters were designed so that balancing has priority over centering. The switching between balancing and centering was realized by adjusting the dynamic importance degrees according to control.

In some efforts, some learning mechanisms and fuzzy controllers are combined to utilize the learning ability and the rule-based knowledge of the fuzzy control. Jun et al. [8] proposed a reinforcement learning algorithm more similar to the higher animal's psychological learning. The algorithm used a fuzzy inference engine and dynamic recurrent neural networks. The algorithm was applied to the cartpole system where 3 input variables, the angular position, the angular velocity of the pole, and the action network's output were used to generate internal reinforcement according to the 63 fuzzy rules. However, the system performance for hard initial configuration of the cartpole system was questionable. Kwon 
[9] proposed a fuzzy BOXES scheme for cartpole control where the BOXES was used to coordinate two fuzzy controllers. The coordination was based on the evaluation of the outcomes of the control action by either of the fuzzy controllers. Unfortunately the control scheme was affected due to the error in the system modeling.

Besides the cartpole system, a couple of fuzzy controllers were used in some applications. In presenting a traffic signal control model [10], the model was composed of two fuzzy controllers. The first level fuzzy controller yields the urgent degree of every traffic phase and the second level fuzzy controller takes the urgent degree as input to give out a Boolean decision value to tell whether the traffic situation is heavy or light. Liu et al. [11] proposed a novel control strategy to improve the realization of the optimal and cooperative control by traditional PID controller for a high power oil transfer system. The control strategy relied on two fuzzy controllers defined by two different input variables respectively and the control variable was formulated by blending the outputs of the two fuzzy controllers.

In this paper, the previous work [12] is expanded to make the control scheme more effective in terms of balancing with less oscillations and centering quickly. For the two fuzzy controllers, each controller is defined, respectively, by 2 input variables, one defined by the angular position and the angular velocity of the pole and the other by the position and the velocity of the cart. The cooperation scheme between the two controllers is due to the observation of the pole configuration in order to use one controller for balancing the pole and the other for centering the cart. The behavior of the cartpole system is discussed in order to evaluate the performance of the proposed control scheme.

\section{Two Fuzzy Controllers}

Although there may be a few cases of using 4 state variables for the fuzzy logic controller [13] for the cartpole system, most fuzzy controllers for the pole balancing problem use 2 state variables, the pole angle $\phi$ and the angular velocity of the pole $\dot{\phi}$. Unfortunately, with these controllers, there is no way to control the position of the cart while the pole is balanced. Moreover, the other 2 state variables, the position of the cart $x$ and the linear velocity of the cart $\dot{x}$, are seldom used as a set of input variables for a fuzzy controller.

First of all, $\phi$-fuzzy controller using 2 state variables, $\phi$ and $\dot{\phi}$ is discussed. To design the $\phi$-fuzzy controller, for example, 3 membership functions are assigned for each input state variable and 7 membership functions for the output variable, force $F$ as shown in Figure 2. Triangular membership functions are used for all membership functions. "Center of Gravity" (COG) defuzzification method [14] is used to combine the recommendations represented by the implied fuzzy sets from 9 rules of Table 1.

The entries, for example, 'NL', 'NM, 'PM', 'PL' in Table 1 indicate the membership functions 'NL', 'NM', 'PM', 'PL' of the output variable $F$ in Figure 2. Table 1 represents the rules based not only on the output values due to [15] but also on the following abstract knowledge: with no information about the motion of the cart, suppose the cart is stationary. If the pole initially inclined to the left $(\phi: N)$ begins to incline to the left further with negative angular velocity $(\dot{\phi}: \mathrm{N})$, the pole hinge should be quickly moved to the left by applying a large negative $(F: \mathrm{NL}$ ) force so that the pole will be prevented from falling down to the left. This describes the reason for the entry 'NL' in the Table 1. If the pole begins to incline to the left from its neutral position $(\phi: Z)$ with negative angular velocity $(\dot{\phi}$ : $\mathrm{N}$ ), the pole hinge should be carefully moved to the left by applying a negative $(F: \mathrm{N})$ force. This describes the reason for the entry ' $\mathrm{N}$ ' $(\phi: \mathrm{Z}$ and $\dot{\phi}: \mathrm{N})$ in the Table 1 . The other entries can be determined by similar reasoning.

There are 9 points on the axis of $F$ in Figure 2. For example, the membership function labeled by 'PM' is a triangle denoted by three points $F_{1}, F_{2}$ and $F_{3}$ on the axis. For every point, $\Delta F=F_{i}-F_{i-1}$ where $i=4,3, \ldots,-3$.

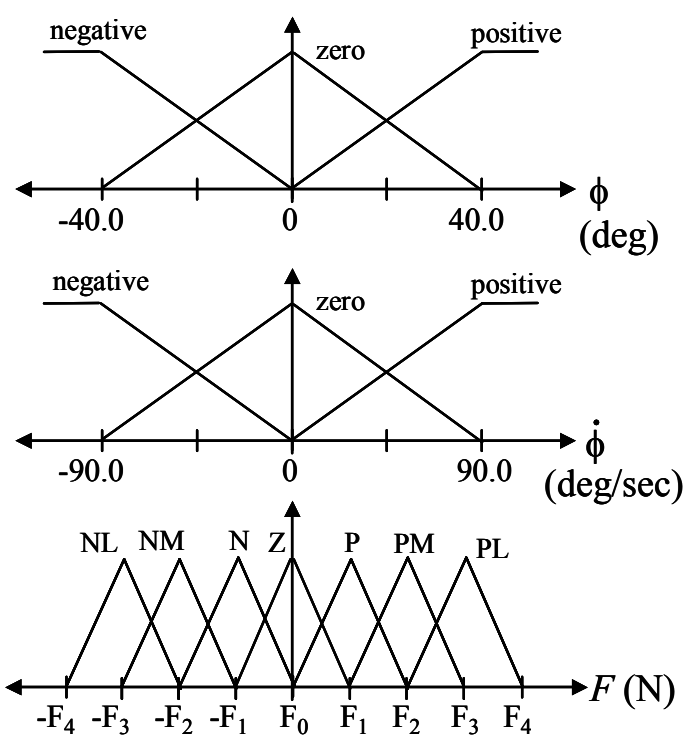

Figure 2. 3 membership functions of $\phi$ and $\phi$ and 7 membership functions of $F$ for the $\phi$-fuzzy controller.

Table 1. Rule table with 9 rules for $F$ with $\phi$ and $\dot{\phi}$.

\begin{tabular}{|c|c|c|c|c|}
\hline \multicolumn{2}{|c|}{$F$} & \multicolumn{3}{|c|}{$\dot{\phi}$} \\
\cline { 3 - 5 } \multicolumn{2}{|c|}{} & $\mathrm{N}$ & $\mathrm{Z}$ & $\mathrm{P}$ \\
\hline \multirow{3}{*}{$\phi$} & $\mathrm{N}$ & $\mathrm{NL}$ & $\mathrm{NM}$ & $\mathrm{N}$ \\
\cline { 2 - 5 } & $\mathrm{Z}$ & $\mathrm{N}$ & $\mathrm{Z}$ & $\mathrm{P}$ \\
\cline { 2 - 5 } & $\mathrm{P}$ & $\mathrm{P}$ & $\mathrm{PM}$ & $\mathrm{PL}$ \\
\hline
\end{tabular}

For a computer simulation for the operation of the cartpole system by the $\phi$-fuzzy controller, the 4 th order Runge-Kutta method with a time step of 0.02 seconds was used to approximate the solution of dynamics equations. The equations 
of the cartpole system and the cartpole parameters are as following [7]:

$$
\begin{gathered}
\ddot{\phi}=\frac{\left(m_{c}+m\right) g \sin \phi-\left(F+m l \dot{\phi}^{2} \sin \phi\right) \cos \phi}{\left\{\frac{4}{3}\left(m_{c}+m\right)-m(\cos \phi)^{2}\right\}} \\
\ddot{x}=\frac{\frac{4}{3}\left(F+m l \dot{\phi}^{2} \sin \phi\right)-m g \sin \phi \cos \phi}{\left\{\frac{4}{3}\left(m_{c}+m\right)-m(\cos \phi)^{2}\right\}}
\end{gathered}
$$

where $g=9.8\left(\mathrm{~m} / \mathrm{sec}^{2}\right) \quad$ is the gravity acceleration, $m=0.1(\mathrm{~kg})$ is the mass of the pole, $m_{c}=1.0(\mathrm{~kg})$ is the mass of the cart, $l=0.5(m)$ is the half of the pole length, $F$ (Newtons) is the force applied to the cart center.

Figure 3 shows the cart position and pole angle versus time when the cartpole system operates by the $\phi$-fuzzy controller with $\Delta F=100 / 4$ and $F_{4}=100$ for the membership functions of $F$ in Figure 2 after the system is released at the state of $(0$, $0,10,0)[16]$. Although the cart keeps on moving away from the track center, it is obvious that the controller is able to balance the pole very quickly.

When $\Delta F$ with $F_{4}$ whose value is smaller than 100 is used for the membership functions of $F$ in Figure 2, the cart moves faster. Then, it is observed that the bigger force $F_{4}$ for the $\phi$-fuzzy controller could move the cart more slowly along the track while keeping the pole balanced. Therefore, it is certain that the $\phi$-fuzzy controller can balance the pole as long as the pole is not very inclined initially, for example, no more than 45 degrees. Also, comparing the performance depicted by Figure 3 with one by [16] where the pole is balanced with a little oscillation in about 1 second appreciates the controller design with the membership functions of Figure 2 and the rule table of Table 1.

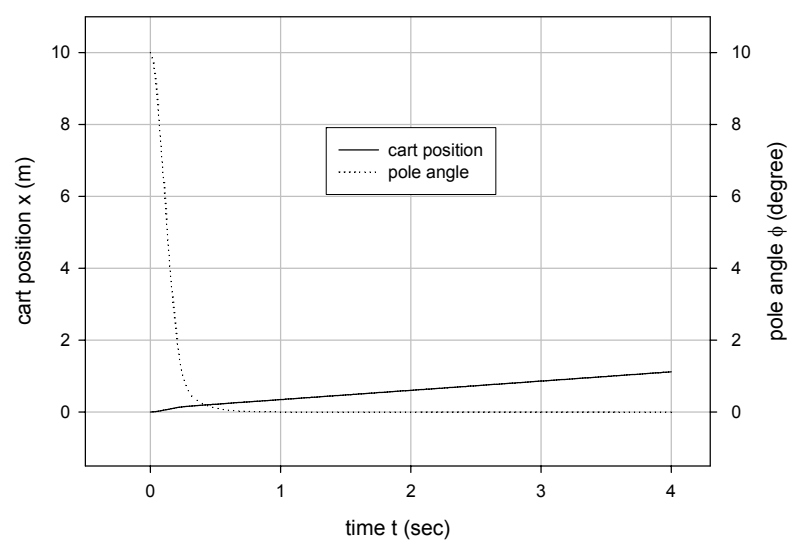

Figure 3. Cart position and pole angle versus time for the $\phi$ fuzzy controller.

To the $\phi$-fuzzy controller, the position and the velocity of the cart are not concerned. Then it is plausible that for the $\phi$ fuzzy controller any state is considered to be $(0,0, \phi, \dot{\phi})$. When the pole is almost balanced, the system state can be described approximately by $(0,0,0,0)$ to the $\phi$-fuzzy controller. Hence the state of the cartpole system is to be approximated by $(x, \dot{x}, 0,0)$ somewhere on the track while the pole is near upright position.

It is possible for the state $(x, \dot{x}, 0,0)$ to be described by only two variables $x$ and $\dot{x}$ while the other two variables $\phi$ and $\dot{\phi}$ can be considered as the parameters of which values are constant, 0 . Then, the steady state of the cartpole system can be partially described by only two state variables $x$ and $\dot{x}$. Due to this observation, the inputs of a fuzzy controller can be composed by these two variables as long as the pole is very near upright .

Now, the second fuzzy controller ( $x$-fuzzy controller) is defined by two input variables $x$ and $\dot{x}$ and one output variable, $F$. Figure 4 shows the membership functions for the variables. There are 11 points on the axis of $F$. For example, the membership function labeled by 'PS' is a triangle denoted by three points $F_{1}, F_{2}$ and $F_{3}$ on the axis.

Table 2. Rule table with 9 rules for $F$ with $x$ and $\dot{x}$.

\begin{tabular}{|c|c|c|c|c|}
\hline \multicolumn{2}{|c|}{$F$} & \multicolumn{3}{|c|}{$\dot{x}$} \\
\cline { 3 - 5 } \multicolumn{2}{|c|}{} & $\mathrm{N}$ & $\mathrm{Z}$ & $\mathrm{P}$ \\
\hline \multirow{3}{*}{$x$} & $\mathrm{~N}$ & $\mathrm{NL}$ & $\mathrm{N}$ & $\mathrm{PS}$ \\
\cline { 2 - 5 } & $\mathrm{Z}$ & $\mathrm{NM}$ & $\mathrm{Z}$ & $\mathrm{PM}$ \\
\cline { 2 - 5 } & $\mathrm{P}$ & $\mathrm{NS}$ & $\mathrm{P}$ & $\mathrm{PL}$ \\
\hline
\end{tabular}
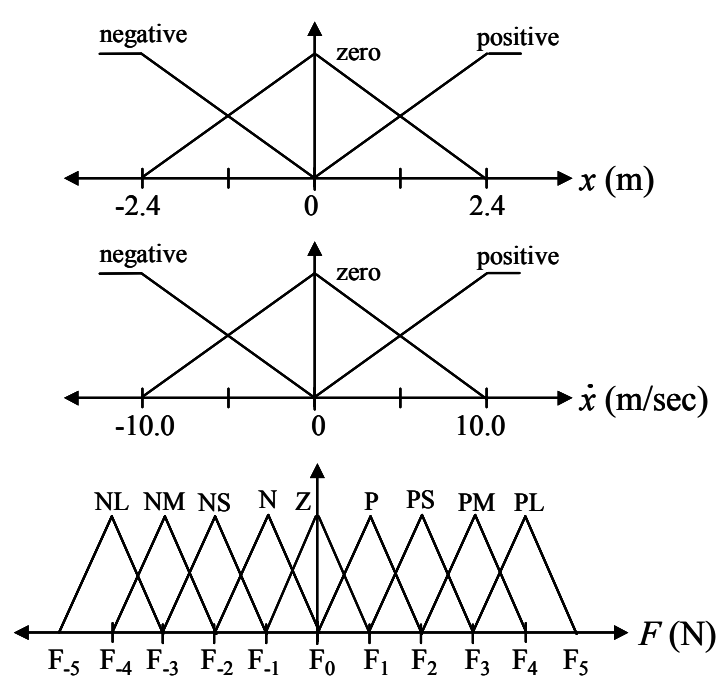

Figure 4. The membership functions of $x, \dot{x}$ and $F$ of the $x$-fuzzy controller.

Since the $x$-fuzzy controller can do nothing alone, the system behavior by the controller is not tried. However, it is expected that the $x$-fuzzy controller should play a role when it is cooperated with the $\phi$-fuzzy controller by a proper scheme. 


\section{Alternation of the Two Fuzzy Controllers}

It is clear that the $\phi$-fuzzy controller has no control over the position of the cart on the track while it is very quick to make the pole balance. On the other hand, one hopes that the $x$ fuzzy controller would assist the $\phi$-fuzzy controller to move the cart toward the track center while the $\phi$-fuzzy controller cares the balancing of the pole. Hence, it is necessary to develop a control scheme that directs the two controllers so that they can accomplish the control in terms of balancing the pole as well as centering the cart of the cartpole system.

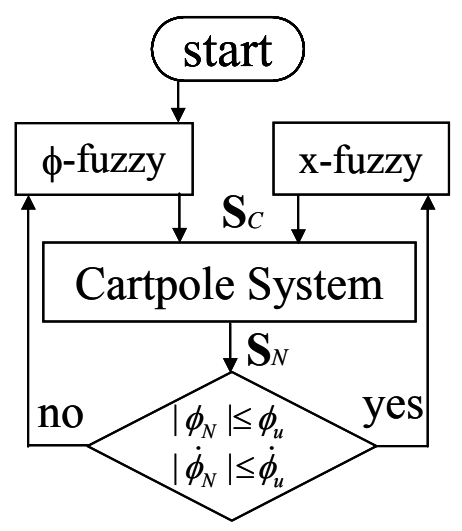

Figure 5. A control scheme for alternating two fuzzy controllers

A simple control scheme is proposed to accomplish the control objective. Figure 5 describes the scheme. Let $S_{c}$ be the state vector for the current system state and $s_{v}$ be the new system state resulting from a control action for $s_{c}$ by either of the controllers. For an initial system state $s_{c}$, the $\phi$-fuzzy controller applies its output force to the cart. Then the system state is denoted by $s_{v}$. IF $\left|\phi_{N}\right| \leq \phi_{u}$ and $\left|\dot{\phi}_{N}\right| \leq \dot{\phi}_{u}$, the $\phi$ fuzzy controller stops working and the $x$-fuzzy controller applies the output force to the cart. Otherwise, the system keeps on working with the $\phi$-fuzzy controller in order to get the pole balanced. Here, $\phi_{u}$ and $\dot{\phi}_{u}$ are the prescribed boundary values of the angle and angular velocity of the pole by which the pole configuration is considered to be in near the upright position.

Decision-making mechanism for alternating two fuzzy controllers is different from that of [12]. For the mechanism, the scheme uses two parameters, $\phi_{u}$ and $\dot{\phi}_{u}$ while [12] uses only $\phi_{u}$. Then, $\left|\phi_{N}\right|$ and $\phi_{u}$ as well as $\left|\dot{\phi}_{N}\right|$ and $\dot{\phi}_{u}$ are compared for the decision. The modification brings forth an improvement on the control performance. This will be discussed later.

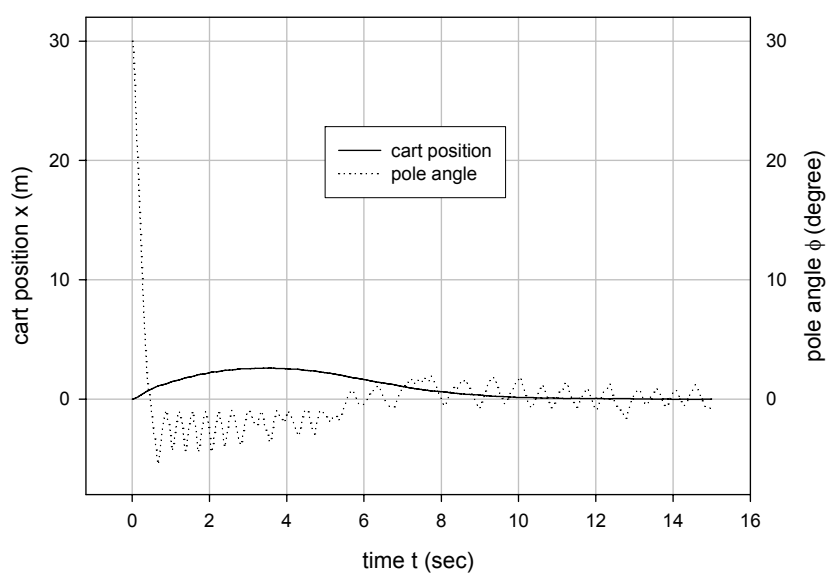

Figure 6. Cart position and pole angle versus time when the system is controlled by a cooperation of two fuzzy controllers with $\phi_{u}=5.0$ and $\dot{\phi}_{u}=10.0$. (For the $x$-fuzzy controller,

$$
\left.\Delta F=100 / 5 \text { and } F_{s}=100\right)
$$

Figure 6 shows the changes of $x$ and $\phi$ versus $t$ after the cartpole system is released at $(0,0,30,0)$ [7] when the proposed control scheme is with $\phi_{u}=5.0$ and $\dot{\phi}_{u}=10.0$. For the $\phi$-fuzzy controller, $\Delta F$ is $100 / 4$ with $F_{4}=100$ in Figure 2. For the $x$-fuzzy controller, the maximum output is the same magnitude as the $\phi$-fuzzy controller. So, $\Delta F$ is $100 / 5$ with $F_{5}=100$ in Figure 4. For the first 0.44 seconds, the $\phi$-fuzzy controller works to make the pole balance. Then, the $x$-fuzzy controller begins to act and increases the number of its action times continuously until the cart reverses its moving direction at $2.6(\mathrm{~m})$ position around 5 seconds. Then the $x$ fuzzy controller becomes the leading controller for the rest of time. As the cart stays around the track center, the $\phi$-fuzzy controller only acts when the pole is about to incline with the angular speed bigger than $\dot{\phi}_{u}=10.0$. Once the cart arrives at the track center at 13.5 second, two controllers cooperate so that the cart moves little and the pole oscillates with the amplitude less than 1.6 degrees.

Comparing Figure 6 with Figure 3 makes the performance of the $x$-fuzzy controller due to the control scheme appreciate. It is clear that the $x$-fuzzy controller plays the role of keeping the cart around the track center while the $\phi$-fuzzy controller does its job for making the pole balance. Thus, one may see that the $\phi$-fuzzy controller takes care of balancing the pole in order for the $x$-fuzzy controller to do its job of keeping the cart around the track center.

Figure 7 shows the force acting by the two fuzzy controllers for Figure 6. The graph shows most of the continuous force acting by the $\phi$-fuzzy controller although it is hard to see the $x$-fuzzy controller acting depicted by dotted line. While the cart moves away from the track center, the $\phi$-fuzzy controller works more frequently than the $x$-fuzzy controller. Once the cart changes its moving direction in 3.34 seconds, the $x$-fuzzy controller works more frequently than before. Due to Figure 7 , two controllers alternate most frequently for the period from 
3.34 seconds to 5.24 seconds. After 5.24 seconds, the $x$-fuzzy controller works more frequently than the $\phi$-fuzzy controller. This situation continues after the cart arrives at the track center at 10.5 seconds. After 10.5 seconds, it is observed that the $\phi$ fuzzy controller works only instantly in order to change the direction of the pole angle such that the pole inclines toward the track center.

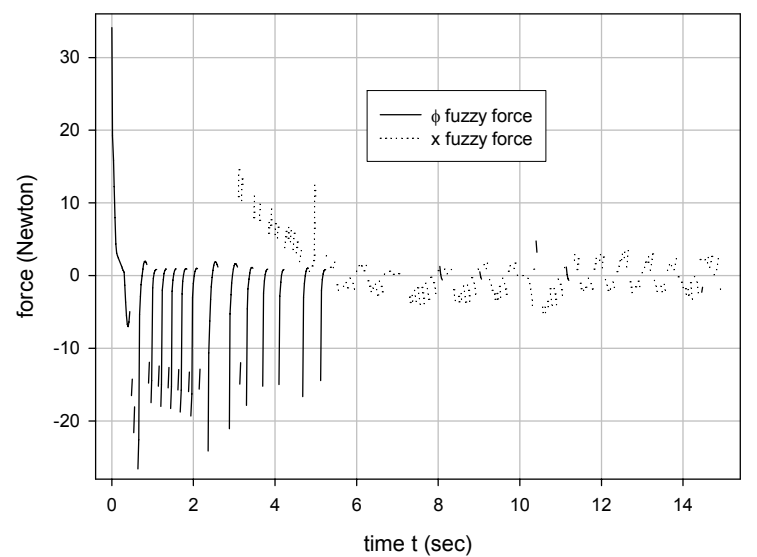

Figure 7. Force acting by the two fuzzy controllers for Figure 6

The control scheme is now tested for the initial state $(1.0,1.0,6.0,12.0)$ [1]. Figure 8 shows the changes in 4 state variables versus $t$. As contrasted with Figure 6, in order to show the minute variation of the cart position, the pole angle in right vertical axis is measured in radian in this figure. The cart keeps moving away from the position of $1.0(\mathrm{~m})$ before it reverses its moving direction at $3.55(\mathrm{~m})$ in 3.44 seconds. About at 13.58 seconds, the cart is back to the track center. Then the pole begins oscillating with the amplitude of 0.03 radians while the cart moves back and forth about the track center.

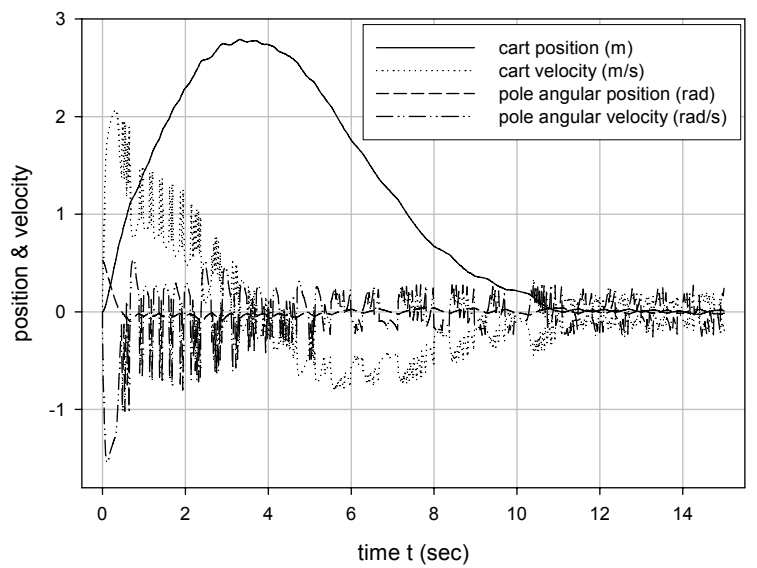

Figure 8. Changes in 4 state variables versus time when the system is controlled by a cooperation of two fuzzy controllers with $\phi_{u}=5.0$ and $\dot{\phi}_{u}=10.0$ after it is released at $(1.0,1.0,6.0$, 12.0)
For Figure 8, the graph of force acting is very similar to Figure 6. It was found that two controllers alternated most frequently for the period from 3 seconds to 5 seconds. One can see that, for the period, the cart velocity keeps on decreasing while it seems that the pole angular speed begins decreasing. There is no special variation of changes in 4 state variables while the switching of two controllers is most frequent. In addition, while the cart and the pole are near the target regulation position after 11 seconds, the cart velocity and the pole angular velocity oscillate within a boundary of maximum $0.25 \mathrm{~m} / \mathrm{sec}$ or $0.3 \mathrm{rad} / \mathrm{sec}$. Also, it seems that the $\phi$-fuzzy controller is to be involved in decreasing both the cart velocity and the pole angular velocity. Then, it is clear that the effect of acting the $\phi$-fuzzy controller on the cart velocity and the pole angular velocity is significant for the first about 3 seconds when the system is quite away from the target regulation position.

\section{Results and Discussion}

According to Figure 6 and Figure 8, the performance of the proposed control scheme is not good in terms of both the time required to stabilize the system and the residual oscillations. Figure 9 shows the changes of $x$ and $\phi$ versus $t$ when the system is controlled by a nonlinear control law [15] after the system is released at $(0,0,30,0)$. It takes less than 10 seconds for the law to do the job with little oscillation. Moreover, by [7], it takes only 8 seconds to stabilize the system. However, the control system is quite complex so that it might require much computational cost. On the other hand, the proposed control scheme is based on just two fuzzy controllers with 9 rules for each controller that are defined by only 2 state variables respectively. Also, the simplicity of the control scheme should be appreciated. Most of all, using the state variables $x$ and $\dot{x}$ as a set of input variables for a fuzzy controller of the cartpole system is a feature of the proposed control scheme.

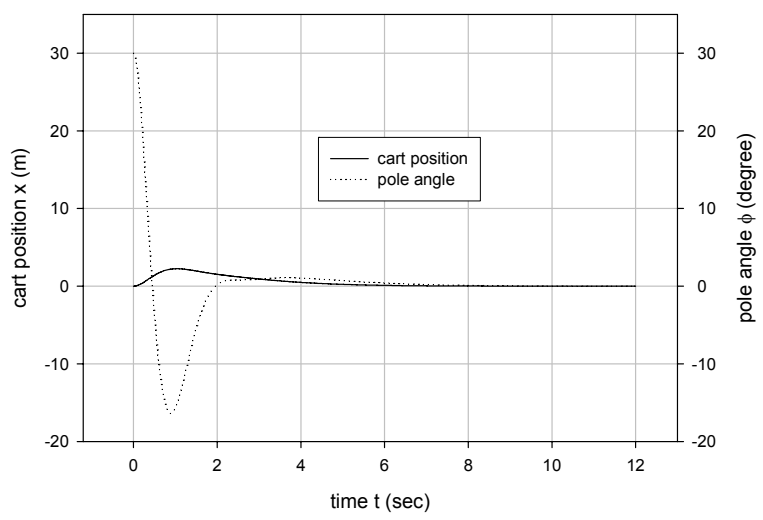

Figure 9. Cart position and pole angle versus time when the system is controlled by a nonlinear control law [15]. 


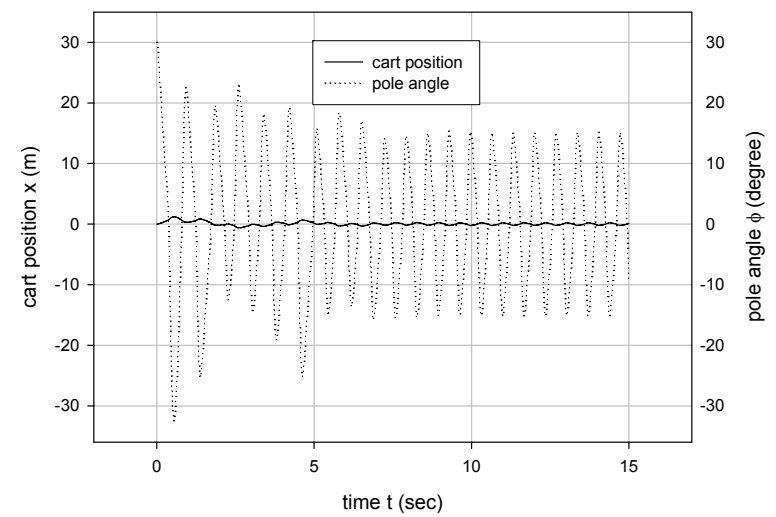

Figure 10. Cart position and pole angle versus time when the system is controlled by a cooperation of two fuzzy controllers of [12].

Figure 10 shows the changes of $x$ and $\phi$ versus $t$ of the cartpole system when the control scheme is due to [12]. The amplitude of the pole oscillation is about 15 degrees as the cart moves $0.2(\mathrm{~m})$ back and forth from the track center. The control scheme of [12] has the same parameters as that of Figure 5 except $\dot{\phi}_{u}$. However, the control performance is much different between Figure 6 and Figure 9. It is obvious that addition of the condition $\left|\dot{\phi}_{N}\right| \leq \dot{\phi}_{u}$ on the decision-making mechanism improves greatly the control performance in terms of the residual oscillations of the pole and the cart.

With various experiments it is found that, with a bigger value of $\phi_{u}$, the cart changes its moving direction in less time and returns to the track center more rapidly, although the pole oscillates with bigger amplitude. This is due to more actions by the $x$-fuzzy controller since the controller will have more time to move the cart owing to the bigger $\phi_{u}$. This observation goes well along with the fact that the $x$-fuzzy controller is to bring the cart back to the track center while the $\phi$-fuzzy controller cares the pole balancing.

The bigger value $\phi_{u}$ is, the more times the $x$-fuzzy controller acts. However, if the value of $\phi_{u}$ becomes bigger than some boundary value, the assumption that the pole is near upright position for the $x$-fuzzy controller can be ineffective. Therefore, it is necessary to be aware of the magnitude of $\phi_{u}$ such that the $x$-fuzzy controller is effective in moving the cart back to the track center.

With a smaller value of $\dot{\phi}_{u}$, the pole oscillates with less amplitude while it takes a longer time before the cart moves back to the track center. Once the cart arrives at the track center, the value of $\dot{\phi}_{u}$ has influence on the angular amplitude of the pole. While the cart moves back and forth about the track center, the alternation is mostly decided by the condition $\left|\dot{\phi}_{N}\right| \leq \dot{\phi}_{u}$ to activate the $x$-fuzzy controller when the pole oscillates with a bigger $\dot{\phi}_{u}$. Of course, the condition $\left|\phi_{N}\right| \leq \phi_{u}$ plays the role of making the cart move toward the track center.
It is estimated that the proposed control scheme is simple enough to be realized by an experiment set up. The 0.02 seconds of sampling time, memory requirement for two 2-D fuzzy controllers with 9 rules respectively and computation speed are all easily adopted for usual PC environment [17].

Here, it is noted that both fuzzy controllers are not defined by all of the 4 state variables. The $\phi$-fuzzy controller is defined by $\phi$ and $\dot{\phi}$ and the $x$-fuzzy controller by $x$ and $\dot{x}$. Thus, producing the output of each of the fuzzy controller gets involved with a half of the numbers of the state variables of the cartpole system. The output application is directed by an alternation of the two fuzzy controllers. The alternation is based on a simple scheme that observes $\phi_{N}$ as well as $\dot{\phi}_{N}$ and selects one of the two fuzzy controllers to produce a proper control action by seeing if $\left|\phi_{N}\right| \leq \phi_{u}$ and $\left|\dot{\phi}_{N}\right| \leq \dot{\phi}_{u}$.

In addition, the $\phi$-fuzzy controller is very sensitive to the rule table configuration while the $x$-fuzzy controller is not much sensitive. When some of the output membership functions in Table 1 are altered, the performance of the controller is greatly changed. Then the rule map of the $\phi$ fuzzy controller is a factor that affects the performance of the proposed control scheme.

\section{Conclusions}

A control scheme making use of two fuzzy controllers is proposed to control the cartpole system. Each of the controllers is defined by two input variables; the $\phi$-fuzzy controller is by $\phi$ and $\dot{\phi}$ and the $x$-fuzzy controller is by $x$ and $\dot{x}$. The two fuzzy controllers alternate to apply the proper control force to the cart by some turns. The control turn alternates due to the decision for the pole configuration that could stay near upright position. Even though the rule set of the $\phi$-fuzzy controller is essential to the performance of control, the control scheme works well to balance the pole as well as to bring the cart back to the track center.

There may be some training algorithms for some intelligent control elements such as CMAC [18], neural networks, and others that will implement the proposed control scheme. Also, those control elements may be able to be defined by 4 or less than 4 state variables to utilize the input-output relationship of the proposed control scheme. Then the intelligent control element will learn the process of alternation of the two fuzzy controllers so that it can direct the fuzzy controllers by the system state. Or some intelligent control element might be able to learn how to adjust the value of $\phi_{u}$ due to the system behavior. The integration of the intelligent control element would reduce the time required for stabilization of the cartpole system. 


\section{References}

[1] Geva, S. and Sitte, J. "A Cartpole Experiment Benchmark for Trainable Controllers," IEEE Control Systems Magazine, Vol. 13, No. 5, pp. 40-51, 1993.

[2] Lee, C.-C., "A Self-Learning Rule-Based Controller Employing Approximate Reasoning and Neural Net Concepts, "International Journal of Intelligent Systems, Vol. 6, pp. 71-93, 1991.

[3] Deng, Z., Zhang, Z., and Jia, P., "A Neural-Fuzzy BOXES Control System with Reinforcement Learning and its Application to Inverted Pendulum," IEEE International Conference on Intelligent Systems for the 21st Century, Vol. 2, pp. 1250-1254, Oct. 22-25 1995.

[4] Michie, D. and Chambers, R. A., '"BOXES' as a Model of Pattern-Formation," in Towards a Theoretical Biology, Vol. 1, Prolegomena, C. H. Waddington, Ed., Edinburgh: Edinburgh Univ. Press, pp. 206-215, 1968.

[5] Lin, C. and Sheu, Y., "A Hybrid-Control Approach for Pendulum-Car Control," IEEE Transactions on Industrial Electronics, Vol. 39, No. 3, pp. 208-214, 1992.

[6] Li, T. and Shieh, M., "Switching-type Fuzzy Sliding Mode Control of a Cart-Pole System," Mechatronics, Vol. 10, pp. 91-109, 2000.

[7] Yi, J. and Yubazaki, N., "Stabilization Fuzzy Control of Inverted Pendulum Systems," Artificial Intelligence in Engineering, Vol. 14, pp. 153-163, 2000.

[8] Jun, H. B., Lee, D. W., Kim, D. J. and Sim, K. B., "Fuzzy Inference-based Reinforcement Learning of Dynamic Recurrent Neural Networks," Proceedings of the 36th SICE Annual Conference, International Session papers, pp. 10831088, 29-31 July 1997.

[9] Kwon, S., "BOXES-based Cooperative Fuzzy Control for Cartpole System," International Journal of Fuzzy Logic and Intelligent Systems, Vol. 7, No. 1, pp. 22-29, March 2007.

[10]Wei, H., Yong, W., Xuanqin, M., and Yan, W., "A Cooperative Fuzzy Control Method for Traffic Lights," 2001 IEEE Intelligent Transportation Systems Conference Proceedings, pp. 185-188, 25-29 August 2001.
[11]Liu, J., Liu, Ding., Zhang, G.-G., and Wu, P.-S., "An Optimal Design of Fuzzy Controller for High Power Oil Transfer System," Proceedings of the First International Conference on Machine Learning and Cybernetics, pp. 699-704, 4-5 November 2002.

[12]Kwon, S., "Two Alternate Fuzzy Controllers for Cartpole System," Proceedings of Sixth International Conference on Machine Learning Cybernetics, Hong Kong, pp. 623-630, 1922 August 2007.

[13]C. L. Karr, Practical Applications of Computational Intelligence for Adaptive Control, CRC Press, pp. 29-40, 1999.

[14] Passino, K. M., Biomimicry for Optimization, Control, and Automation, Springer-Verlag London Limited, pp. 222-223, 2005.

[15]Guez, A. and Selinsky J., "A Trainable Neuromorphic Controller," Journal of Robotic Systems, Vol. 5, No. 4, pp. 363-388, 1988.

[16] Jang, J. R., "Self-Learning Fuzzy Controllers Based on Temporal Back Propagation," IEEE Transactions on Neural Networks, Vol. 3, No. 5, pp. 714-723, September 1992.

[17] Yamakawa, T., "A Fuzzy Inference Engine in Nonlinear Analog Mode and Its Application to a Fuzzy Logic Control," IEEE Transactions on Neural Networks, Vol. 4, No. 3, pp. 496-522, May 1993.

[18]J. S. Albus, "A New Approach to Manipulator Control; The Cerebellar Model Articulation Controller (CMAC)," Journal of Dynamic Systems, Measurement, and Control, Transactions of the ASME, Series G, Vol. 97, No. 3, pp. 220-227, September 1975

Sunggyu Kwon received the B.S. and M.S. degrees in the Department of Mechanical Engineering, Yonsei University, Korea, in 1980 and 1983 respectively, and Ph.D. degree in the Department of Mechanical Engineering, Louisiana State University in 1990. He worked as a Senior Researcher in the Department of Remote Technology Development, Korea Advanced Energy Research Institute from 1991 to 1994. Since 1995, he has been a faculty of the School of Mechanical and Automotive Engineering, Keimyung University. His current research interests include CMAC applications for robotic engineering and fuzzy control issues. 